\title{
Review Article \\ T-Cell Costimulatory Molecules in Acute-Graft-Versus Host Disease: Therapeutic Implications
}

\author{
Javier Briones, Silvana Novelli, and Jorge Sierra \\ Hematology Service, Hospital Santa Creu i Sant Pau, Mas Casanovas 90, 08041 Barcelona, Spain \\ Correspondence should be addressed to Javier Briones, jbriones@santpau.cat
}

Received 26 July 2010; Accepted 20 August 2010

Academic Editor: Philip L. McCarthy

Copyright () 2011 Javier Briones et al. This is an open access article distributed under the Creative Commons Attribution License, which permits unrestricted use, distribution, and reproduction in any medium, provided the original work is properly cited.

Acute Graft-versus-host disease (GVHD) is a major complication after allogeneic hematopoietic stem cell transplantation. Although this process is thought to consist of several phases, T-cell activation plays a critical role in the pathogenesis of acute GVHD. To become efficient effectors, T-cells require additional costimulation after T-cell receptor signaling. A number of molecules are involved in costimulation of T-cells such as CD28, CD40L, CD30, OX40, 4-1BB, ICOS, and LIGHT. The system is regulated by inhibitory molecules, CTLA-4, and PD-1. There is experimental evidence that those molecules are implicated in the pathogenesis of GHVD. We describe how these molecules are involved in acute GVHD and how the blockade of costimulatory molecules may have potential implications for the treatment of patients with acute GVHD.

\section{Introduction}

Acute graft-versus-host disease (GVHD) remains the most important cause of morbidity and mortality after allogeneic hematopoietic stem cell transplantation [1]. The recent advances in the pathogenesis of this complication have not been fully translated into an improved treatment for the patients. Only a few immunosuppressive agents are available for the treatment of acute GVHD and thus, new, more selective treatments are needed.

Acute GVHD is an immune-mediated disease that results from a complex interaction between immune cells from both, the donor, and recipient. The pathophysiology of this process is thought to consist of several phases: (1) priming of the immune response through the conditioning treatments that induce inflammation, and secondary activation of antigen presenting cells (APCs) and T-cells, (2) activation of T-cells which leads to an expansion of effector cells, and finally, (3) trafficking of activated T-cells to target tissues where inflammation and tissue destruction occurs [1].

Donor-derived T-cells are considered to be the main effector cells mediating acute GVHD. Donor T-cells are able to recognize HLA disparities between the donor and recipient, which is directly related to the development of acute GVHD. Furthermore, recipients of HLA identical transplants can still develop acute GVHD due to differences in the so-called minor histocompatibility antigens [2].

$\mathrm{T}$-cell activation in response to an alloantigen requires two stimulatory signals [3]. The first signal happens through the T-cell receptor (TCR), which recognizes the antigen and is HLA restricted. This signal is necessary but not sufficient to induce a full T-cell activation. Signal 2, known as costimulation, is necessary to induce T-cell proliferation, cytokine secretion, and effector function after TCR activation. This costimulation signal is mediated by a number of molecules mostly expressed on APCs that include the B7-CD28 family, the TNF receptor family, and adhesion molecules such as LFA-1. In addition, the costimulatory system is tightly regulated by a subset of inhibitory molecules such as cytotoxic T-lymphocyte antigen 4 (CTLA-4) and programmed death-1 (PD-1) [4] (Table 1). Costimulatory signals are essential for the majority of T-cell functions; in the absence of a proper costimulation T-cells become unresponsive or die because of apoptosis. Studies performed in experimental models of acute GVHD have shown that costimulatory molecules play a pivotal role in the initiation of acute GVHD [5]. This paper will focus upon the role of T-cell costimulatory molecules in GVHD and how these data could be translated into the development of new therapies for patients with acute GVHD. 
TABLE 1: T-cell costimulatory/inhibitory molecules and their ligands.

\begin{tabular}{lccc}
\hline Molecules & Cell expression & Ligand & Cell expression \\
\hline Stimulatory & & & APCs \\
CD28 & T-cells & CD80 (B7-1) & APCs \\
CD40 & CD86 (B7-2) & CD40L (CD154) & T-cells $\left(\mathrm{CD} 4^{+}\right)$ \\
OX40 (CD134) & T-cells & OX40L & APCs \\
$4-1 B B(C D 137)$ & T, NK cells & 4-1BBL & APCs \\
ICOS & T-cells & ICOSL (B7h) & APCs \\
LIGHT & T, NK cells & HVEM & T-cells, APCs \\
CD30 & T, NK, B-cells & CD30L (CD153) & T-cells (CD4 $4^{+}$ \\
\hline Inhibitory & & CD 80, CD86 & APCs \\
CTLA-4 (CD152) & T-cells & PDL1 (B7H1) & DCs, T-cells \\
PD-1 & T-cells & PDL2 (B7DC) & Monocytes \\
\hline
\end{tabular}

APCs: antigen presenting cells.

\section{B7/CD28/CTLA-4 Pathway}

The CD28 receptor and its ligands, the B7 family, are the first signaling pathway necessary for T-cell costimulation [6]. CD28 is a receptor constitutively expressed on T-cells (both $\mathrm{CD}^{+}$and $\mathrm{CD}^{+}$) and NK cells. Ligation of CD28 after TCR signaling enhances T-cell proliferation and cytokine secretion. The main ligands of CD28 are the B7 family, with B7-1 (CD80) and B7-2 (CD86) being the two most important molecules. CD80 and CD86 are expressed on APCs, mostly B-cells and dendritic cells, and the expression is induced after those cells are activated. Experimental studies have shown that proinflammatory signals generated following the conditioning treatment upregulate CD80 and CD86 expression in GVHD target tissues, establishing the rationale for the CD28/B7 pathway blockade in the GVHD treatment.

Studies in GVHD animal models have demonstrated the potential utility of antibodies blocking B7-1 and B7-2 in acute GVHD. Animals receiving both anti-CD80 and CD86 antibodies experienced lower mortality due to acute GVHD, and this was mediated by inhibition of donor $\mathrm{CD}^{+}$and $\mathrm{CD}^{+} \mathrm{T}$-cell expansion $[7,8]$. Those studies also showed that expression of B7-1 on donor $\mathrm{CD}^{+}{ }^{+} \mathrm{T}$-cells was critical for GVHD development, and hence, the treatment with antiB7 antibodies also contributed to reduce GVHD not only by targeting B7 expression on the APCs but also by a direct effect on $\mathrm{CD}^{+}{ }^{+} \mathrm{T}$-cells. However, GVHD treatment based upon B7 blockade was far from perfect and other studies focused on CD28. By using CD28-deficient mice models it has been shown that T-cells were able to induce less severe GVHD, providing evidence that GVHD depends, at least partially, on signaling through CD28 [9]. In this scenario, selective targeting of CD28 with a blocking antibody inhibits T-cell donor expansion and significantly prevents GVHD in murine models. More importantly, inhibition of GVHD with anti-CD28 proved to be superior to the treatment with antiB7 antibodies. To add complexity to the involvement of the CD28 pathway in GVHD, recent studies have focused upon the use of superagonistic anti-CD28 antibodies [10]. These molecules reduce GVHD mostly by preferential targeting of regulatory T-cells (Tregs) over conventional T-cells, thus preserving the graft versus tumor reaction (GVT) [11]. A combination of CD28 stimulation plus rapamycin was also shown to prevent acute GVHD in animal models, confirming previous data suggesting that, at least in mice, Tregs require CD28 costimulation to maintain their suppressive functions [12].

CTLA-4 is a molecule that acts along the CD28-B7 stimulation pathway by inhibiting T-cell alloreactivity [4]. Once T-cells are activated, CTLA-4 is upregulated and binds to $\mathrm{B} 7$ proteins (CD80 and CD86) with higher affinity than CD28, thus resulting in inhibition of T-cell activation. A strategy using CTLA-4 to inhibit GVHD has been tested in animal models. Infusion of CTLA4-Ig (a soluble fusion protein containing the extracellular domain of CTLA4 linked to an IgG Fc region) was able to reduce GVHD in recipients of mismatched grafts [13]. However, inhibition of GVHD was not complete and, comparatively, its inhibitory effect on GVHD was lesser than using anti-CD28 antibodies [8].

The clinical translation of the knowledge of the CD28B7-CTLA-4 pathway to the treatment of GHVD in humans has been scarce, partially because of the high complexity of this system. Nevertheless, the proof of concept has been demonstrated in a selected group of patients $(n=12)$ receiving a haploidentical marrow graft [14]. To reduce the elevated risk of acute GVHD in these patients, the donor marrow was cocultured ex vivo with irradiated mononuclear cells from the patient in the presence of CTLA4-Ig. The rationale of this approach was to induce anergy of the alloreactive donor T-cells, while preserving the GVT effect. Only three patients developed acute GVHD, which was successfully treated with steroids in two of them.

A new CTLA4-Ig fusion protein derivative, Abatacept, inhibits the interaction of $\mathrm{B} 7$ proteins with $\mathrm{CD} 28$, and reduces INF- $\gamma$ and IL-17 secretion. While it is being used for treating autoimmune diseases, its role in the treatment of acute GVHD has not been tested so far [15]. 


\section{CD40/CD40L Pathway}

The CD40-CD40L pathway plays a critical role in the regulation of both the humoral and cellular immune response [16]. CD40, a member of the tumor necrosis factor receptor (TNFR) superfamily, is expressed on APCs (B-cells, dendritic cells and monocytes), and its ligand, CD40L (CD154), is expressed on activated $\mathrm{CD}^{+} \mathrm{T}$-cells and a small subset of CD8 ${ }^{+}$T-cells. Ligation of CD40 stimulates the expression of costimulatory molecules on APCs (i.e. CD80, CD86) and contributes to an effective activation of T-cells. In addition, CD40-CD40L interactions enhance the secretion of proinflammatory cytokines (i.e., IL-2, IL-12, and IFN- $\gamma$ ) that further contributes to the activation of T and NK cells. Ex vivo blockade of $\mathrm{CD} 4^{+} \mathrm{T}$-cells using anti-CD40L antibody induces tolerance of $\mathrm{CD}^{+} \mathrm{T}$-cells to host alloantigens and reduces GVHD, while preserving $\mathrm{T}$-cell responses directed to those antigens not present during tolerisation [17].

The role of this system in the pathogenesis of GVHD was demonstrated in experimental GVHD models, where the administration of anti-CD40L antibodies was able to reduce the lethality of GVHD [18]. When pure T-cell populations were administered into sublethally irradiated recipients, anti-CD40L antibodies improved the GHVD caused by $\mathrm{CD} 4^{+} \mathrm{T}$-cells, but not by $\mathrm{CD} 8^{+} \mathrm{T}$-cells, consisting with the preferential expression of $\mathrm{CD} 40 \mathrm{~L}$ on activated $\mathrm{CD} 4^{+} \mathrm{T}$-cells [19]. However, mechanistic studies on the action of antiCD40L antibodies do not support the idea that it only affects T-cell costimulation. In fact, anti-CD40L antibodies seem to act primarily through the selective depletion of activated Tcells, rather than costimulatory blockade [20]. Interestingly, several studies have shown that blockade of CD40-CD40L pathway induces Tregs that may contribute to the GVHD inhibition [21,22].

Studies comparing the blockade of CD40L versus CD28 pathway have shown that the use of anti-CD40L was significantly more effective than anti-B-7 antibodies in reducing GVHD [23]. In addition, experimental studies suggest that dual blockade of CD40L and CD28 pathway may be useful in reducing GVHD lethality. In this regard, a selective B7CD28 blockade, using CTLA4-Ig plus anti-CD40L blockade have been shown to reduce GVHD severity and may be more advantageous than using anti-CD28 antibodies [22].

Another important issue, when using a costimulatory blockade to improve GVHD, is the preservation of the GVT effect. In this regard, treatment with anti-CD40L had a deleterious effect on the GVT reaction in a murine allogeneic hematopoietic transplantation model, in contrast to antibodies blocking CD28. The inhibition of the GVT effect may be due to the inhibitory effects on Th1 and cytotoxic T-cells and inhibition of IL-12 cytokine secretion [24].

\section{OX40/OX40L Pathway}

OX40 (CD134) is a member of the TNFR family and is expressed on activated $\mathrm{CD} 4^{+}$and $\mathrm{CD} 8^{+} \mathrm{T}$-cells. Its ligand, OX40L, is expressed preferentially on APCs, that is, dendritic cells and B-cells. Signaling through OX40 induces generates costimulatory signals resulting in cytokine secretion, Th1 and Th2, and promotes T-cell proliferation and development into memory cells [25].

Studies done in animal models have shown that OX40OX40L interactions play an important role in the pathogenesis of GVHD [26, 27]. T-cells expressing OX40 were present in lymphohematopoietic organs of rats with GVHD, and $\mathrm{CD}^{+}$and $\mathrm{CD} 8^{+} \mathrm{T}$-cells from mice with GVHD showed upregulation of OX40.

In the clinical setting, studies performed in patients undergoing allogeneic stem cell transplantation (SCT) have showed an involvement of OX40 in the development of GVHD. Thus, in our experience, among a series of activating and costimulatory T-cell molecules, OX40 was preferentially upregulated in $\mathrm{CD} 4^{+} \mathrm{T}$-cells from patients with acute GVHD [28]. Other studies have also shown the existence of OX40expressing T-cells in patients with acute and chronic GVHD $[29,30]$.

The increased presence of $\mathrm{OX} 40^{+} \mathrm{T}$-cells in GVHD models leads to studying how OX40-OX40L blockade could affect GVHD. The administration of a blocking antibody against OX40L reduced the GVHD-induced mortality in irradiated mice receiving MHC-disparate T-cells [27]. In contrast, agonistic anti-OX40 antibody markedly increased the mortality in the same model. These results are consistent with the data showing that OX40-OX40L interactions clearly accelerate the GVHD when it is preferentially mediated by $\mathrm{CD} 4^{+}$T-cells.

In humans, in vitro depletion of alloreactive $\mathrm{OX} 40^{+} \mathrm{T}-$ cells resulted in a T-cell population that has reduced alloantigen-specific reactivity while it has retained Tcell specificity against third-party antigens including virus (CMV) and tumor antigens (WT1) [31]. Collectively, all the preclinical data suggest that interrupting the OX40-OX40L pathway may be an interesting strategy to modulate GVHD in patients undergoing allogeneic SCT.

\section{4-1BB/4-1BBL Pathway}

4-1BB (CD137) is a member of the TNFR superfamily with important implications in T-cell costimulation [25]. 4-1 $\mathrm{BB}$ is rapidly expressed on activated $\mathrm{CD} 4^{+}$and $\mathrm{CD} 8^{+}$ T-cells. Its ligand, 4-1BBL, is expressed on APCs, that is, dendritic cells, B-cells, and macrophages. CD40 is a major regulator of $4-1 \mathrm{BB}$ expression on APCs. When the signaling through the TCR is strong, $4-1 \mathrm{BB} / 4-1 \mathrm{BBL}$ interaction provides costimulatory signals to T-cells, independently of CD28. Although, in vitro, $4-1 \mathrm{BB}$ can activate both $\mathrm{CD} 4^{+}$ and $\mathrm{CD} 8^{+} \mathrm{T}$-cells, $4-1 \mathrm{BB}$ agonistic antibodies preferentially act on $\mathrm{CD} 8^{+} \mathrm{T}$-cells. $4-1 \mathrm{BB}$ interaction induces proliferation and expansion of $\mathrm{CD}^{+}$T-cells, upregulation of effector molecules including perforin and granzyme A, and IL-2 and INF- $\gamma$ secretion. In addition, 4-1BB prevents T-cell apoptosis and prolongs T-cell survival. The importance of 4$1 \mathrm{BB}$ interactions in regulating graft-versus-host reactions has been shown in studies performed in MHC class II disparate hosts receiving $\mathrm{CD} 4^{+} \mathrm{T}$-cells, where the administration of an agonistic anti $4-1 \mathrm{BB}$ antibody induces $100 \%$ mortality, as a resultant of a severe GVHD. In contrast, when the recipients 
are $4-1 \mathrm{BB}$ negative GVHD lethality is largely reduced [32].

With these data, several groups have explored the potential effect of 4-1BB blockade in the development of GVHD. In an acute GVHD murine model, the administration of an anti-4-1BBL antibody reduced GVHD lethality, and this was associated with a diminished donor $\mathrm{CD} 8^{+} \mathrm{T}$-cell expansion [33]. In line with these data, treatment of donor T-cells with anti-4-1BBL antibody reduced GVHD and improved survival over a standard cyclosporin A + methotrexate combination in a MHC disparate GVHD model [34]. Collectively, the experimental data indicate that $4-1 \mathrm{BB} / 4$ $1 \mathrm{BBL}$ blockade can contribute to significantively reduce GVHD.

\section{ICOS/ICOS-L Pathway}

The inducible costimulator (ICOS) is a member of the CD28 family, and is expressed in activated $\mathrm{CD} 4^{+}$and $\mathrm{CD} 8^{+} \mathrm{T}$-cells [35]. Its ligand, ICOSL, also known as B7h, is expressed on Bcells, dendritic cells and macrophages. ICOSL is upregulated by inflammatory cytokines such as INF- $\gamma$ and TNF- $\alpha$. ICOS interaction preferentially regulates $\mathrm{Th} 2$ cytokine production (IL-4 and IL-10), specially in the presence of CD28 signaling [36]. ICOS interaction is responsible for T-cell expansion and the effector phase of the immune response, in a CD28independent fashion. Although the effect of blocking ICOS interaction was shown preferentially in chronic GHVD models, consistent with its effects on Th2 differentiation [37], studies done in fully allograft GVHD models have revealed a prominent role in the development of acute GVHD [23]. Thus, administration of an anti-ICOS antibody at the time of the SCT was very effective in reducing GVHD and improving survival in an acute GVHD model. In line with these data, studies performed in several murine acute GVHD models have definitively shown that ICOS blockade inhibits the expansion of alloreactive T-cells, and strongly reduced the number of effector cells, preferentially in critical GHVD target tissues such as skin and intestine, which suggest that ICOS may also be involved in the regulation of T-cell homing and homeostasis [38]. A fully human antibody blocking human ICOS has been developed and proved to ameliorate GVHD in severe immunodeficient mice grafted with human blood mononuclear cells [39]. Given the nonredundant roles of the CD28 and ICOS pathway in T-cell costimulation, strategies combining ICOS blockade plus CD28 blockade may constitute an attractive strategy for GHVD modulation in the clinical scenario [40].

\section{LIGHT/HVEM Pathway}

LIGHT, a member of the TNF superfamily and homologous to lymphotoxins, is involved in T-cell costimulation and antigen presentation by dendritic cells [25]. LIGHT is expressed upon activation of $\mathrm{CD}^{+}$and $\mathrm{CD}^{+}$T-cells, in NK cells, and is also detected in immature dendritic cells. Although it has been described in three different receptors, the primary receptor for LIGHT in T-cells is HVEM (Herpes virus entry mediator), which is expressed in resting T-cells, NK cells, and dendritic cells. LIGHT provides a costimulatory signal on Tcells, which is independent of the CD28 pathway, enhances T-cell proliferation and Th1 cytokine secretion. In addition to its effects on T-cells, LIGHT contributes to dendritic cell maturation, enhanced by CD40L, which results in further Tcell activation.

Several studies have suggested a role for LIGHT in alloreactivity. In an allogeneic mixed lymphocyte reaction LIGHT interactions enhances T-cell proliferation, while the absence of LIGHT, in both responders and stimulators, results in inhibition of proliferation [41]. The involvement of LIGHT in GVHD has been demonstrated in preclinical studies showing that blockade of LIGHT with a soluble receptor was able to reduce GVHD and improve survival [42]. However, this protective effect was only detected when GVHD was preferentially mediated by $\mathrm{CD}^{+} \mathrm{T}$-cells, while completely failed when the model was dependant on $\mathrm{CD} 4^{+}$ T-cells. Since acute GVHD in humans is mediated by both $\mathrm{CD}^{+}$and $\mathrm{CD}^{+}$T-cells, investigators tested the blockade of LIGHT combined with CD40L blockade in a murine GVHD model [43]. Administration of a LIGHT soluble receptor plus anti-CD40L antibody beginning on day 0 of the SCT completely prevented acute GVHD and improved survival. Although this strategy induced a profound state of tolerance, the fact that alloantigen-specific T-cells were rendered anergic complicates its translation to the clinical scenario due to the lost of the GVT effect.

\section{CD30/CD30L Pathway}

CD30, a member of the TNFR superfamily, is expressed on activated T-cells, NK cells, and B-cells [25]. Its ligand, CD30L (CD153), is expressed on resting B-cells and activated T-cells. Engagement of CD30 in the presence of TCR stimulation enhances T-cell proliferation and cytokine production. Although initial analysis suggested a role for CD30 on Th2 development, studies have shown a role of CD30 as a T-cell costimulatory molecule involved in both Th1 and Th2 immune response [44]. CD30L induces proliferation of T-cells when they are previously treated with agonistic anti-CD3 antibodies. CD30L is also found in areas of T-cell and B-cell contact, and provides signals for B-cell growth and differentiation. CD30-deficient mice have an increased autoreactivity, and in humans, alloreactive $\mathrm{T}$-cells preferentially reside within the $\mathrm{CD} 30^{+} \mathrm{T}$-cell subset.

The involvement of the CD30 system in GVHD has been demonstrated in experimental models. Mice receiving CD30negative MHC class II disparate T-cells had longer survival compared to animals receiving wild-type $\left(\mathrm{CD} 30^{+}\right) \mathrm{T}$-cells. Consisting with this data, administration of a blocking antiCD30L antibody improved survival in mice after receiving MHC disparate CD4 ${ }^{+}$T-cells [45].

\section{PD-1/PD-1L Pathway}

Programmed death-1 (PD-1), a member of the immunoglobulin superfamily, is expressed on activated T-cells, B-cells, and myeloid cells. Experimental data indicates that 
PD-1 is a co-inhibitor of T- and B-cell responses [46]. Two ligands have been shown to interact with PD-1: PD-L1, also known as B7-H1, is a member of the B7 family, and is expressed on dendritic cells and activated T-cells; PD-L2, also termed B7-DC, and is expressed on resting monocytes. Both ligands are expressed in some nonlymphohematopoietic tissues.

In vivo studies have shown that the main role of $\mathrm{PD}-1$ is to inhibit T-cell responses [47]. Studies to address if this molecule had a role in the pathogenesis of acute GVHD showed an increase in PD-1 infiltrating cells in GVHD target organs (intestine, liver) in murine models receiving full MHC disparate T-cells. PD-1 blockade by administration of anti-PD-1 antibodies resulted in acceleration of GVHD and enhanced mortality, mostly mediated by IFN- $\gamma$ secretion from donor T-cells [48]. In line with this, experimental studies using T-cells from PD-1 knockout mice have revealed an enhanced capacity to induce GVHD. Thus, the PD-1/PD-L pathway seems to be an important mediator of GVHD. Collectively, these data suggest that agonistic antibodies to $\mathrm{PD}-1$ may represent a novel strategy for preventing GVHD.

\section{Conclusions}

T-cell costimulatory molecules play a critical role in the development of acute GVHD. In addition to the extensively studied CD28, other molecules have been ultimately implicated in GVHD. In particular, several members of the TNFR family (CD40, OX-40, 4-1BB) have relevant roles in T-cell activation and contribute to the development of acute GVHD in humans; monoclonal antibodies directed to these molecules are being developed to use in the clinical setting. A critical issue is to develop therapeutic strategies that, while blocking alloreactivity, do not compromise the GVT effect. In line with this, the blockade of critical Tcell costimulatory molecules such as CD40L and 4-1BBL, although potentially useful for reducing GVHD severity, may severely compromise the GVT effect which may preclude their use in the clinical practice. However, the fact that the blockade of molecules such as OX-40L did not significantly affect the GVT in experimental models, and in vitro depletion of $\mathrm{OX} 40 \mathrm{~L}^{+} \mathrm{T}$-cells did not affect to thirdparty allospecific T-cells makes this molecule an interesting target for prevention or treatment of acute GVHD. On the other hand, the better understanding of the mechanism of molecules involved in counter regulation of T-cell activation (i.e., CTLA-4 and PD-1) makes these molecules attractive targets for GVHD therapy. It is likely that a CTLA-4 fusion protein will be tested in patients with acute GVHD soon, as it is already used in patients with autoimmune diseases.

In conclusion, in the next years new monoclonal antibodies and inhibitors of critical costimulatory molecules will increase the therapeutic strategies for the prevention or treatment of patients with acute GVHD after allogeneic stem cell transplantation. It is expected that these new therapeutic strategies will help to reduce the mortality of this complication.

\section{References}

[1] J. L. Ferrara, J. E. Levine, P. Reddy, and E. Holler, "Graftversus-host disease," Lancet, vol. 373, no. 9674, pp. 1550-1561, 2009.

[2] J. M. M. den Haan, N. E. Sherman, E. Blokland et al., "Identification of a graft versus host disease-associated human minor histocompatibility antigen," Science, vol. 268, no. 5216, pp. 1476-1480, 1995.

[3] A. G. Baxter and P. D. Hodgkin, "Activation rules: the two-signal theories of immune activation," Nature Reviews Immunology, vol. 2, no. 6, pp. 439-446, 2002.

[4] M.-L. Alegre, K. A. Frauwirth, and C. B. Thompson, "T-cell regulation by CD28 and CTLA-4," Nature Reviews Immunology, vol. 1, no. 3, pp. 220-228, 2001.

[5] X. C. Li, D. M. Rothstein, and M. H. Sayegh, "Costimulatory pathways in transplantation: challenges and new developments," Immunological Reviews, vol. 229, no. 1, pp. 271-293, 2009.

[6] A. H. Sharpe and G. J. Freeman, "The B7-CD28 superfamily," Nature Reviews Immunology, vol. 2, no. 2, pp. 116-126, 2002.

[7] B. R. Blazar, A. H. Sharpe, P. A. Taylor et al., "Infusion of antiB7.1 (CD80) and anti-B7.2 (CD86) monoclonal antibodies inhibits murine graft-versus-host disease lethality in part via direct effects on CD4+ and CD8+ T cells," Journal of Immunology, vol. 157, no. 8, pp. 3250-3259, 1996.

[8] X.-Z. Yu, S. J. Bidwell, P. J. Martin, and C. Anasetti, "CD28specific antibody prevents graft-versus-host disease in mice," Journal of Immunology, vol. 164, no. 9, pp. 4564-4568, 2000.

[9] X.-Z. Yu, P. J. Martin, and C. Anasetti, "Role of CD28 in acute graft-versus-host disease,” Blood, vol. 92, no. 8, pp. 2963-2970, 1998.

[10] Y. Kitazawa, M. Fujino, X.-K. Li et al., "Superagonist CD28 antibody preferentially expanded Foxp3-expressing nTreg cells and prevented graft-versus-host diseases," Cell Transplantation, vol. 18, no. 5-6, pp. 627-637, 2009.

[11] N. Beyersdorf, X. Ding, T. Hünig, and T. Kerkau, "Superagonistic CD28 stimulation of allogeneic T cells protects from acute graft-versus-host disease," Blood, vol. 114, no. 20, pp. 4575-4582, 2009.

[12] T. N. Golovina, T. Mikheeva, M. M. Suhoski et al., "CD28 costimulation is essential for human $\mathrm{T}$ regulatory expansion and function," Journal of Immunology, vol. 181, no. 4, pp. 2855-2868, 2008.

[13] P. M. Wallace, J. S. Johnson, J. F. MacMaster, K. A. Kennedy, P. Gladstone, and P. S. Linsley, "CTLA4Ig treatment ameliorates the lethality of murine graft-versus-host disease across major histocompatibility complex barriers," Transplantation, vol. 58, no. 5, pp. 602-610, 1994.

[14] E. C. Guinan, V. A. Boussiotis, D. Neuberg et al., "Transplantation of anergic histoincompatible bone marrow allografts," New England Journal of Medicine, vol. 340, no. 22, pp. 1704$1714,1999$.

[15] A. Beck, T. Wurch, C. Bailly, and N. Corvaia, "Strategies and challenges for the next generation of therapeutic antibodies," Nature Reviews Immunology, vol. 10, no. 5, pp. 345-352, 2010.

[16] J. Banchereau, F. Bazan, D. Blanchard et al., "The CD40 antigen and its ligand," Annual Review of Immunology, vol. 12, pp. 881-922, 1994.

[17] B. R. Blazar, P. A. Taylor, R. J. Noelle, and D. A. Vallera, "CD4+ T cells tolerized ex vivo to host alloantigen by anti-CD40 ligand (CD40L:CD154) antibody lose their graftversus-host disease lethality capacity but retain nominal antigen responses," Journal of Clinical Investigation, vol. 102, no. 3, pp. 473-482, 1998. 
[18] F. H. Durie, A. Aruffo, J. Ledbetter et al., "Antibody to the ligand of CD40, gp39, blocks the occurrence of the acute and chronic forms of graft-vs-host disease," Journal of Clinical Investigation, vol. 94, no. 3, pp. 1333-1338, 1994.

[19] B. R. Blazar, P. A. Taylor, A. Panoskaltsis-Mortari et al., "Blockade of CD40 ligand-CD40 interaction impairs CD4+ T cell-mediated alloreactivity by inhibiting mature donor $\mathrm{T}$ cell expansion and function after bone marrow transplantation," Journal of Immunology, vol. 158, no. 1, pp. 29-39, 1997.

[20] R. E. G. Hargreaves, N. J. Monk, and S. Jurcevic, "Selective depletion of activated T cells: the CD40L-specific antibody experience," Trends in Molecular Medicine, vol. 10, no. 3, pp. 130-135, 2004.

[21] P. A. Taylor, T. M. Friedman, R. Korngold, R. J. Noelle, and B. R. Blazar, "Tolerance induction of alloreactive $\mathrm{T}$ cells via ex vivo blockade of the CD40:CD40L costimulatory pathway results in the generation of a potent immune regulatory cell," Blood, vol. 99, no. 12, pp. 4601-4609, 2002.

[22] B. Verbinnen, A. D. Billiau, J. Vermeiren et al., "Contribution of regulatory $t$ cells and effector $t$ cell deletion in tolerance induction by costimulation blockade," Journal of Immunology, vol. 181, no. 2, pp. 1034-1042, 2008.

[23] B. R. Blazar and P. A. Taylor, "Role of T-cell costimulation and regulatory cells in allogeneic hematopoietic cell transplantation," in Graft-vs-Host Disease, J. Ferrara, K. Cooke, and H. G. Deeg, Eds., pp. 83-124, 2004.

[24] J. Ohata, J. Sakurai, K. Saito, K. Tani, S. Asano, and M. Azuma, "Differential graft-versus-leukaemia effect by CD28 and CD40 co-stimulatory blockade after graft-versus-host disease prophylaxis," Clinical and Experimental Immunology, vol. 129, no. 1, pp. 61-68, 2002.

[25] M. Croft, "Control of immunity by the TNFR-related molecule OX40 (CD134)," Annual Review of Immunology, vol. 28, pp. 57-78, 2010.

[26] T. V. Tittle, A. D. Weinberg, C. N. Steinkeler, and R. T. Maziarz, "Expression of the T-cell activation antigen, OX-40, identifies alloreactive T cells in acute graft-versus-host disease," Blood, vol. 89, no. 12, pp. 4652-4658, 1997.

[27] B. R. Blazar, A. H. Sharpe, A. I. Chen et al., "Ligation of OX40 (CD134) regulates graft-versus-host disease (GVHD) and graft rejection in allogeneic bone marrow transplant recipients," Blood, vol. 101, no. 9, pp. 3741-3748, 2003.

[28] M. Paz Morante, J. Briones, E. Canto et al., "Activationassociated phenotype of CD3+ T cells in acute graft-versushost disease," Clinical and Experimental Immunology, vol. 145, no. 1, pp. 36-43, 2006.

[29] J. Sanchez, J. Casaño, M. A. Alvarez et al., "Kinetic of regulatory CD25high and activated CD134+ (OX40) T lymphocytes during acute and chronic graft-versus-host disease after allogeneic bone marrow transplantation," British Journal of Haematology, vol. 126, no. 5, pp. 697-703, 2004.

[30] L. S. Lamb Jr., S. A. Abhyankar, L. Hazlett et al., "Expression of CD134 (OX-40) on $\mathrm{T}$ cells during the first 100 days following allogeneic bone marrow transplantation as a marker for lymphocyte activation and therapy-resistant graft-versushost disease," Communications in Clinical Cytometry, vol. 38, no. 5, pp. 238-243, 1999.

[31] X. Ge, J. Brown, M. Sykes, and V. A. Boussiotis, "CD134allodepletion allows selective elimination of alloreactive human $\mathrm{T}$ cells without loss of virus-specific and leukemiaspecific effectors," Biology of Blood and Marrow Transplantation, vol. 14, no. 5, pp. 518-530, 2008.
[32] B. R. Blazar, B. S. Kwon, A. Panoskaltsis-Mortari, K. B. Kwak, J. J. Peschon, and P. A. Taylor, "Ligation of 4-1BB (CDw137) regulates graft-versus-host disease, graft-versusleukemia, and graft rejection in allogeneic bone marrow transplant recipients," Journal of Immunology, vol. 166, no. 5, pp. 3174-3183, 2001.

[33] K. Nozawa, J. Ohata, J. Sakurai et al., "Preferential blockade of CD8+ $\mathrm{T}$ cell responses by administration of anti-CD137 ligand monoclonal antibody results in differential effect on development of murine acute and chronic graft-versus-host diseases," Journal of Immunology, vol. 167, no. 9, pp. 49814986, 2001.

[34] K. Xu, C. Li, X. Pan, and B. Du, "Study of relieving graftversus-host disease by blocking CD137-CD137 ligand costimulatory pathway in vitro," International Journal of Hematology, vol. 86, no. 1, pp. 84-90, 2007.

[35] A. Hutloff, A. M. Dittrich, K. C. Beier et al., "ICOS is an inducible T-cell co-stimulator structurally and functionally related to CD28," Nature, vol. 397, no. 6716, pp. 263-266, 1999.

[36] R. A. Shilling, B. S. Clay, A. G. Tesciuba et al., "CD28 and ICOS play complementary non-overlapping roles in the development of Th2 immunity in vivo," Cellular Immunology, vol. 259, no. 2, pp. 177-184, 2009.

[37] S.-H. Ogawa, G. Nagamatsu, M. Watanabe et al., "Opposing effects of anti-activation-inducible lymphocyteimmunomodulatory molecule/inducible costimulator antibody on the development of acute versus chronic graftversus-host disease," Journal of Immunology, vol. 167, no. 10, pp. 5741-5748, 2001.

[38] P. A. Taylor, A. Panoskaltsis-Mortari, G. J. Freeman et al., "Targeting of inducible costimulator (ICOS) expressed on alloreactive $\mathrm{T}$ cells down-regulates graft-versus-host disease (GVHD) and facilitates engraftment of allogeneic bone marrow (BM)," Blood, vol. 105, no. 8, pp. 3372-3380, 2005.

[39] N. Tajima, K. Tezuka, A. Tanimoto et al., "JTA-009, a fully human antibody against human AILIM/ICOS, ameliorates graft-vs-host reaction in SCID mice grafted with human PBMCs," Experimental Hematology, vol. 36, no. 11, pp. 15141523, 2008.

[40] S. A. Nanji, W. W. Hancock, C. C. Anderson et al., "Multiple combination therapies involving blockade of ICOS/B7RP1 costimulation facilitate long-term islet allograft survival," American Journal of Transplantation, vol. 4, no. 4, pp. 526-536, 2004.

[41] K. Tamada, K. Shimozaki, A. I. Chapoval et al., "LIGHT, a TNF-like molecule, costimulates $\mathrm{T}$ cell proliferation and is required for dendritic cell-mediated allogeneic $\mathrm{T}$ cell response," Journal of Immunology, vol. 164, no. 8, pp. 41054110, 2000.

[42] Y. Xu, A. S. Flies, D. B. Flies et al., "Selective targeting of the LIGHT-HVEM costimulatory system for the treatment of graft-versus-host disease," Blood, vol. 109, no. 9, pp. 40974104, 2007.

[43] K. Tamada, H. Tamura, D. Flies et al., "Blockade of LIGHT/LT $\beta$ and CD40 signaling induces allospecific T cell anergy, preventing graft-versus-host disease," Journal of Clinical Investigation, vol. 109, no. 4, pp. 549-557, 2002.

[44] P. Pellegrini, A. M. Berghella, I. Contasta, and D. Adorno, "CD30 antigen: not a physiological marker for TH2 cells but an important costimulator molecule in the regulation of the balance between TH1/TH2 response," Transplant Immunology, vol. 12, no. 1, pp. 49-61, 2003. 
[45] B. R. Blazar, R. B. Levy, T. W. Mak et al., "CD30/CD30 ligand (CD153) interaction regulates CD4+ $\mathrm{T}$ cell-mediated graftversus-host disease," Journal of Immunology, vol. 173, no. 5, pp. 2933-2941, 2004.

[46] L. Chen, "Co-inhibitory molecules of the B7-CD28 family in the control of T-cell immunity," Nature Reviews Immunology, vol. 4, no. 5, pp. 336-347, 2004.

[47] T. Okazaki and T. Honjo, "The PD-1-PD-L pathway in immunological tolerance," Trends in Immunology, vol. 27, no. 4, pp. 195-201, 2006.

[48] B. R. Blazar, B. M. Carreno, A. Panoskaltsis-Mortari et al., "Blockade of programmed death-1 engagement accelerates graft-versus-host disease lethality by an IFN- $\gamma$-dependent mechanism," Journal of Immunology, vol. 171, no. 3, pp. 1272 1277, 2003. 


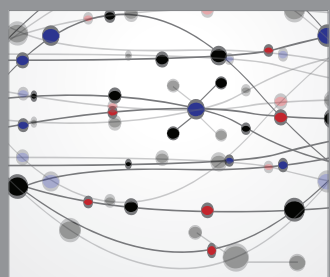

The Scientific World Journal
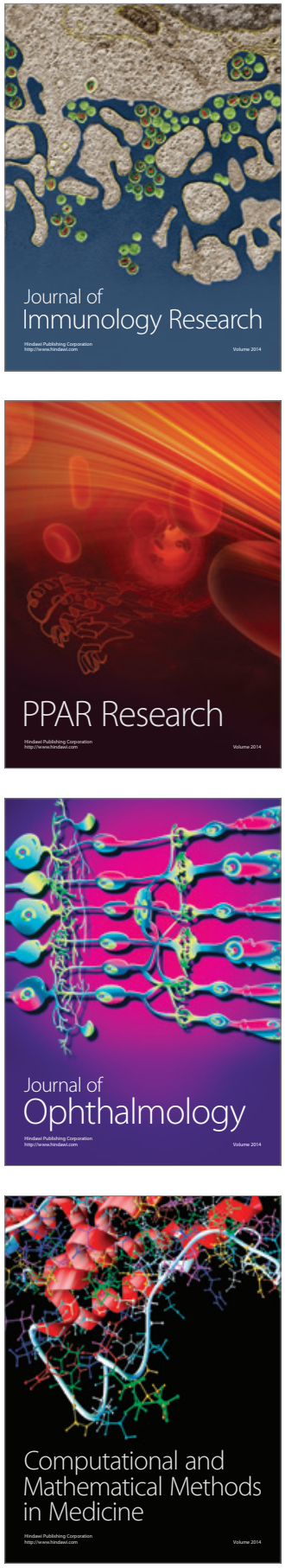

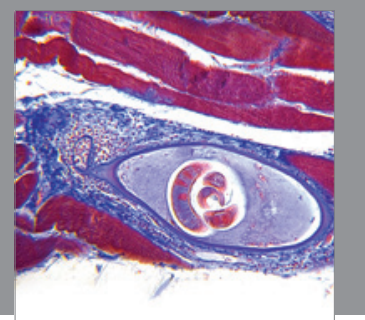

Gastroenterology

Research and Practice
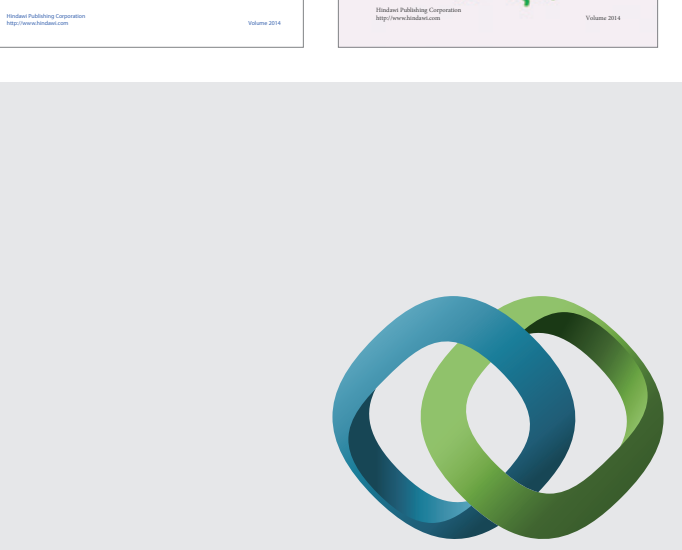

\section{Hindawi}

Submit your manuscripts at

http://www.hindawi.com
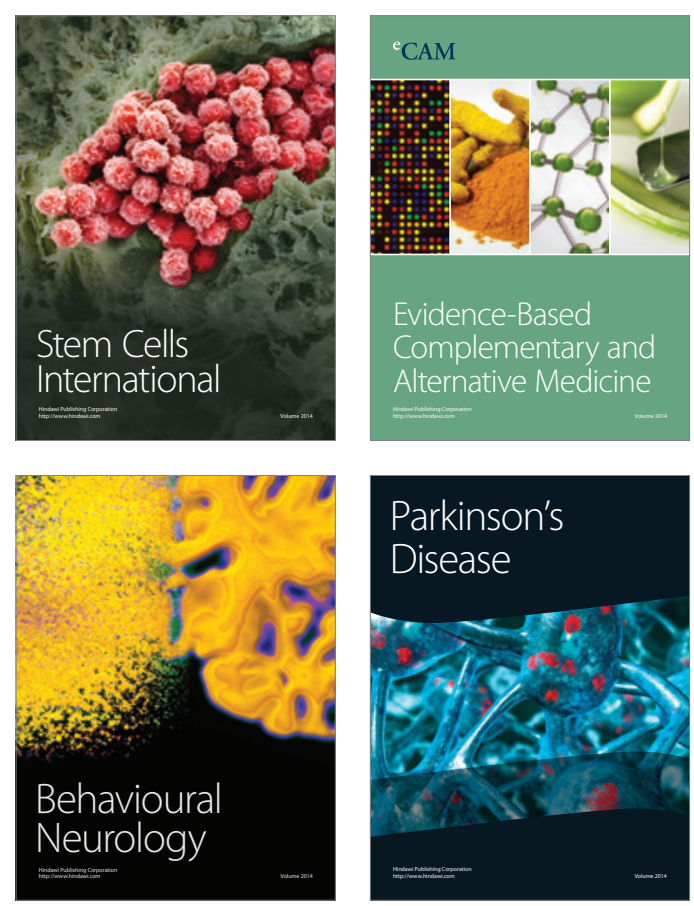

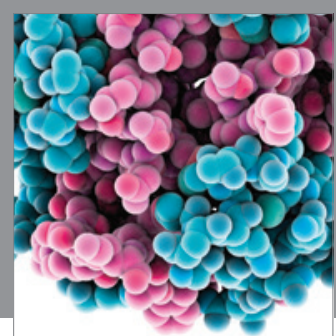

Journal of
Diabetes Research

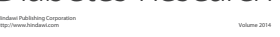

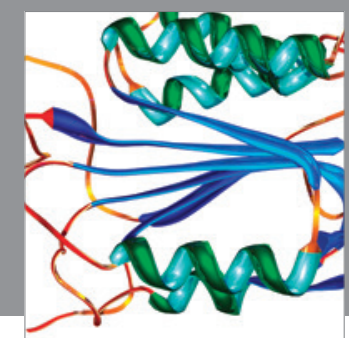

Disease Markers
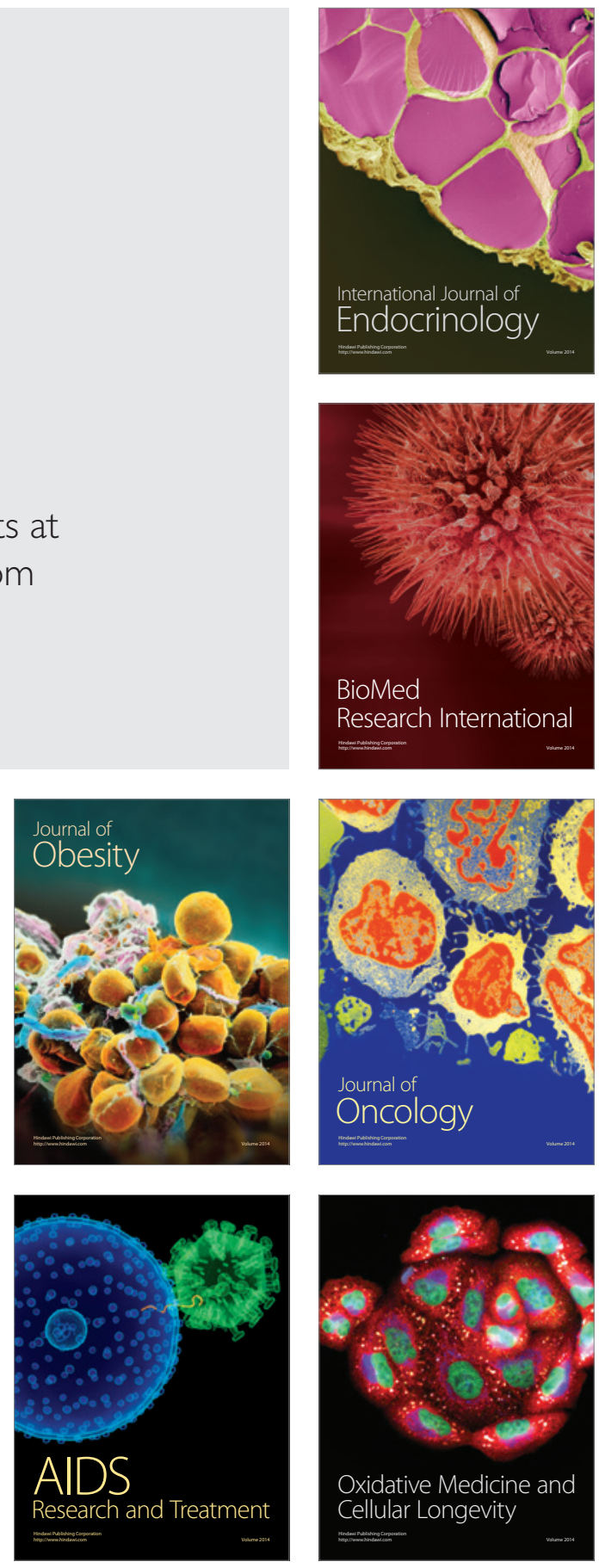